\title{
Performance of the EUDET-type beam telescopes
}

\author{
Hendrik Jansen ${ }^{1 *}$ (D), Simon Spannagel ${ }^{1}$, Jörg Behr ${ }^{1,6}$, Antonio Bulgheroni ${ }^{2,7}$, Gilles Claus ${ }^{3}$, \\ Emlyn Corrin ${ }^{4,8}$, David Cussans ${ }^{5}$, Jan Dreyling-Eschweiler ${ }^{1}$, Doris Eckstein ${ }^{1}$, Thomas Eichhorn ${ }^{1}$, \\ Mathieu Goffe ${ }^{3}$, Ingrid Maria Gregor ${ }^{1}$, Daniel Haas ${ }^{4,9}$, Carsten Muhll ${ }^{1}$, Hanno Perrey ${ }^{1,10}$, \\ Richard Peschke ${ }^{1}$, Philipp Roloff ${ }^{1,11}$, Igor Rubinskiy ${ }^{1,12}$ and Marc Winter ${ }^{3}$
}

*Correspondence: hendrik.jansen@desy.de 'Deutsches Elektronen-Synchrotron DESY, Hamburg, Germany Full list of author information is available at the end of the article

\begin{abstract}
Test beam measurements at the test beam facilities of DESY have been conducted to characterise the performance of the EUDET-type beam telescopes originally developed within the EUDET project. The beam telescopes are equipped with six sensor planes using MIMOSA 26 monolithic active pixel devices. A programmable Trigger Logic Unit provides trigger logic and time stamp information on particle passage. Both data acquisition framework and offline reconstruction software packages are available. User devices are easily integrable into the data acquisition framework via predefined interfaces.

The biased residual distribution is studied as a function of the beam energy, plane spacing and sensor threshold. Its standard deviation at the two centre pixel planes using all six planes for tracking in a $6 \mathrm{GeV}$ electron/positron-beam is measured to be $(2.88 \pm 0.08) \mu \mathrm{m}$. Iterative track fits using the formalism of General Broken Lines are performed to estimate the intrinsic resolution of the individual pixel planes. The mean intrinsic resolution over the six sensors used is found to be (3.24 \pm 0.09$) \mu \mathrm{m}$. With a $5 \mathrm{GeV}$ electron/positron beam, the track resolution halfway between the two inner pixel planes using an equidistant plane spacing of $20 \mathrm{~mm}$ is estimated to $(1.83 \pm 0.03) \mu \mathrm{m}$ assuming the measured intrinsic resolution. Towards lower beam energies the track resolution deteriorates due to increasing multiple scattering. Threshold studies show an optimal working point of the MIMOSA 26 sensors at a sensor threshold of between five and six times their RMS noise. Measurements at different plane spacings are used to calibrate the amount of multiple scattering in the material traversed and allow for corrections to the predicted angular scattering for electron beams.
\end{abstract}

Keywords: Charged particle tracking, Beam telescope, Pixel sensors, General broken lines, Multiple scattering

\section{Introduction}

Beam telescopes are vital tools for R\&D projects focussing on position sensitive particle detection sensors. These range from collider-specific detectors with high radiation tolerance [1, 2], high resolution and low material requirements [3] to medical applications [4], among others. Complementary to sensor simulations using finite element analysis tools, test beam studies are used at various stages of sensor and read-out chip

(c) 2016 The Author(s). licensee Springer on behalf of EPJ. This is an Open Access article distributed under the terms of the Creative Commons Attribution License (http://creativecommons.org/licenses/by/4.0), which permits unrestricted use, distribution, and reproduction in any medium, provided the original work is properly credited. 
development. Such test beam studies are well suited and often used for the evaluation of the performance of a detector prototype.

Within the Integrated Infrastructure Initiative funded by the EU in the 6th Framework Programme, the EUDET project [5] aimed at providing a high-resolution pixel beam telescope for test beam studies [6]. The guidelines for the development were to allow for an easy integration of custom data acquisition systems covering a wide range of readout schemes, latencies, and acquisition rates. This is achieved by well defined interfaces on both the hardware and the software level. Fast LHC-type tracking devices are integrable in the same manner as slower rolling-shutter readout devices.

The EUDET-type beam telescope each consist of six pixel detector planes equipped with fine-pitch MIMOSA 26 sensors [7], the mechanics for precise positioning of the device under test (DUT) and the telescope planes in the beam, a Trigger Logic Unit (TLU) providing trigger capabilities and a data acquisition system. The chosen design meets most user requirements in terms of easy integration capabilities, spatial resolution, and trigger rates. The telescope planes are designed and built to keep the material budget as low as possible in order to achieve an excellent track resolution even at the rather low particle energies of up to $6 \mathrm{GeV}$ at the DESY test beam facilities.

The original EUDET beam telescope, which was modified to become the AIDA telescope, is operated at SPS beamline H6 (CERN). Responding to the increasing demand of the sensor R\&D community, several replicas, collectively called EUDET-type beam telescopes, have been built since then: ACONITE for the ATLAS group, which is also operated at the beamline H6, ANEMONE at ELSA (University of Bonn), the copy for the Carlton University called CALADIUM, and two copies, DATURA and DURANTA, which are operated at DESY. Within the AIDA2020 project, another copy is going to be built - operation is foreseen at the PS beamline (CERN). All replicas are based on the MIMOSA 26 sensors and are equipped with the same data acquisition system and software framework. The EUDET telescope has been used since 2007 in various stages of development by hundreds of users and played an important role in sensor studies employed by a wide community. From January 2013 until March 2014 alone, about 300 users utilised an EUDET-type beam telescope at DESY for a total of 80 user weeks. The results reported here are based on data taken with DATURA at test beam area 21 at DESY-II and are comparable to other beam telescope copies with a similar thickness of the epitaxial layer [8].

This paper is organised as follows: The DESY beamlines are introduced in the "Beamlines" section, followed by the description of the beam telescope and the data acquisition framework in the "Components of the EUDET-type beam telescopes" and "The EUDAQ data acquisition framework" sections, respectively. The "Offline analysis and reconstruction using EUTelescope" section details the offline analysis and reconstruction software. Results of the EUDET-type beam telescope performance and track resolution predictions for different telescope configurations and beam momenta are presented in the "Track resolution studies" section. Additionally, predictions of the standard deviation of the angular scattering distribution are compared to measurements with an electron beam. The "Considerations for DUT integrations" section discusses the integration of DUTs into an EUDET-type beam telescope. 




Fig. 1 The DATURA beam telescope with its sensor planes (green frame) on aluminium rails. The auxiliary boards (red frame) with connections for clock, sensor configuration, and data are mounted the top of the planes. Coolant is provided to the planes via tubes

\section{Beamlines}

For this work, data have been taken at the DESY-II test beam facilities [9]. The DESY-II electron/positron synchrotron at the DESY site in Hamburg has a circumference of $292.8 \mathrm{~m}$ and is mainly used as an injector for the PETRA-III storage ring. However, it also supplies beam to three test beam areas, one of them being the test beam area 21 . Its dipole magnets operate in a sinusoidal ramping mode with a frequency of $12.5 \mathrm{~Hz}$. Therefore, one DESY-II cycle takes $80 \mathrm{~ms}$, and the bunch length is around $30 \mathrm{ps}$. The DESY-II synchrotron is equipped with movable carbon fibres. If positioned in the beam, bremsstrahlung photons are created, escaping the beam line tangentially. Subsequently, the photons are converted to electron/positron pairs on a secondary metal target. Their energy distribution reaches up to $6 \mathrm{GeV}$. Using a dipole magnet, this secondary electron/positron beam is spread out. A collimator can be used to select certain energy ranges of the beams reaching the experimental halls with an achievable rate of about $10 \mathrm{kHz}$ to $100 \mathrm{kHz}$. A more detailed description of the test beams at DESY-II can be found in reference [10].

\section{Components of the EUDET-type beam telescopes}

The EUDET-type beam telescopes are tabletop tracking detectors featuring six pixelated silicon sensors, four scintillators with photo multiplier tubes (PMTs) for trigger purposes, a Trigger Logic Unit (TLU) providing trigger logic and time stamp information on particle passage, and a data acquisition system for the readout. Figure 1 shows the beam telescope with its rectangular aluminium sensor jigs, wherein the pixel sensors are embedded, and its auxiliary boards providing connections for power, sensor configuration, clock signals and data transmission. The planes are organised in two telescope arms holding three sensors each. A DUT can be inserted between the arms or at either end of the beam telescope. In the Cartesian, right-handed coordinate system chosen, the $y$-direction points vertically down and the $z$-direction along the beam direction. 


\section{Sensors and mechanics}

The MIMOSA 26 sensors used for precise spatial measurements of particle trajectories are manufactured with the AMS $350 \mathrm{~nm}$ CMOS technology [7]. Each MIMOSA 26 sensor consists of pixels sized $18.4 \mu \mathrm{m} \times 18.4 \mu \mathrm{m}$, which are arranged in 1152 columns and 576 rows. This adds up to a total of about six hundred thousand readout channels per sensor, covering an active area of about $21.2 \times 10.6 \mathrm{~mm}$. The specifications of the MIMOSA 26 sensors quote a thickness of $50 \mu \mathrm{m}$. Measurements with a digital microscope reveal an average thickness over the six sensors used of $(54.5 \pm 3.6) \mu \mathrm{m}$. Free charge carriers produced in the underlying $20 \mu \mathrm{m}$ high resistivity (about $400 \Omega \mathrm{cm}$ ) epitaxial layer ${ }^{1}$ are collected via drift (diffusion) in depleted (undepleted) regions. The binary resolution of $5.3 \mu \mathrm{m}$ is improved by charge sharing, i.e. the collection of charge at adjacent pixels and subsequent calculation of the centre of gravity, as is shown in the "Track resolution studies" section.

The MIMOSA 26 sensors are read out with a rolling-shutter, taking 16 cycles of an $80 \mathrm{MHz}$ clock per row, with all columns being read out in parallel. This allows for correlated double sampling and zero suppression on-chip with the digital circuitry placed outside the active pixel array. At this clock frequency, the MIMOSA 26 integration time equals $115.2 \mu \mathrm{s}$, allowing for about 8680 frames to be read out per second. The expected maximum rate of detectable particles through the active area is estimated to be about $1 \mathrm{MHz} / \mathrm{cm}^{2}$ due to the limited on-chip buffer size. The detection threshold is programmable via so-called JTAG files. These provide configurations with different threshold levels in integer multiples $\xi_{n}$ of the RMS noise of the individual planes. A sensor threshold setting of $\xi_{\mathrm{n}}=6$ therefore corresponds to a collected charge in a single pixel of at least six times the noise. At this threshold, the average noise occupancy per pixel is measured to be about $6 \cdot 10^{-5}$ per readout frame at room temperature [11].

Every pixel sensor is mounted within an aluminium jig, three jigs are in turn mounted on each of the two aluminium arms. The jigs feature a beam window around the position of the sensor location, minimising the material budget. Lightproof Kapton foils of $25 \mu \mathrm{m}$ thickness protect the sensors on each side. The overall material of the beam telescope thus amounts to $300 \mu \mathrm{m}$ of silicon and $300 \mu \mathrm{m}$ of Kapton. The two telescope arms, usually one up- and one downstream of the DUT, are movable along the direction of the beam in order to allow for variably sized DUTs to be fitted into the set-up. The minimal distance between two sensors is given by the jig thickness of $20 \mathrm{~mm}$, the maximal distance is restricted by the length of the aluminium arms to $150 \mathrm{~mm}$ at equidistant spacing. Furthermore, the jigs are cooled keeping the MIMOSA 26 sensors at a constant temperature of typically $18^{\circ} \mathrm{C}$ for stable operation. The entire beam telescope is placed on a rotatable frame easing adjustment of its orientation parallel to the beam. Additionally, this frame is mounted on a sturdy structure providing stability over time and wheels for easy transportation.

Important parameters are compiled in Fig. 2. The distance between MIMOSA 26 planes is denoted as $\mathrm{d} z$, the distance between the DUT and its nearest neighbouring MIMOSA 26 planes $\mathrm{d} z_{\mathrm{DUT}}$. The $z$-positions of the pixel planes are denoted $z_{0}$ to $z_{5}$ and the DUT is placed at $z_{\mathrm{DUT}}$. The expression $\varepsilon=\sum_{i} x_{i} / X_{0, i}$ defines the material budget of the scattering medium as the physical material thicknesses $x_{i}$ normalised to their radiation lengths, with values of $X_{0}=93.65 \mathrm{~mm}$ for silicon, $X_{0}=3.042 \cdot 10^{5} \mathrm{~mm}$ for dry air, and $X_{0}=285.6 \mathrm{~mm}$ for Kapton, cf. Table III.6 of reference [12]. The material budget of a 


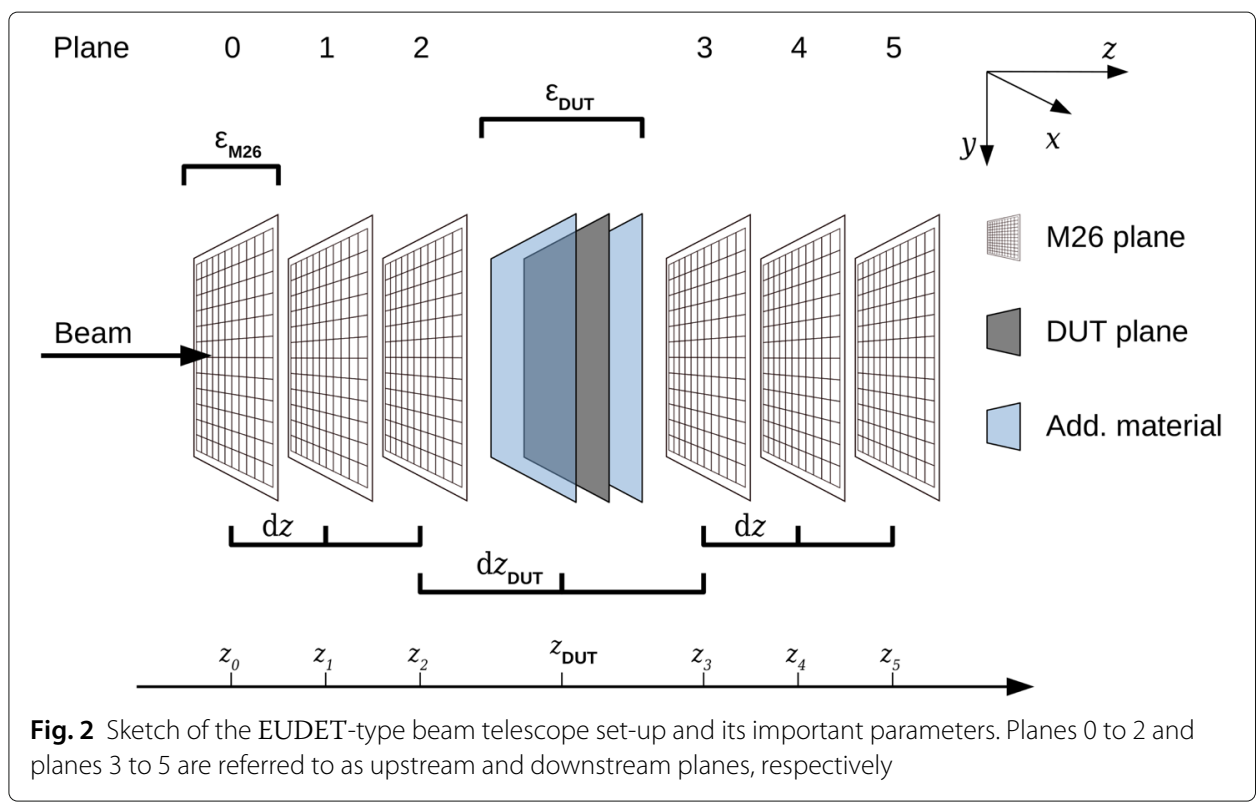

MIMOSA 26 plane including a protecting Kapton foil on each side is $\varepsilon_{\mathrm{M} 26}=7.5 \cdot 10^{-4}$ at an assumed sensor thickness of $54 \mu \mathrm{m}$. At a spacing of $\mathrm{d} z=20 \mathrm{~mm}(\mathrm{~d} z=150 \mathrm{~mm})$ the total material budget amounts to $4.8 \cdot 10^{-3}\left(7.0 \cdot 10^{-3}\right)$. Likewise, $\varepsilon_{\text {DUT }}$ represents the DUT's material budget which includes the DUT itself and other material such as PCBs and cooling boxes.

\section{Trigger and DAQ system}

Four Hamamatsu PMT assemblies with scintillators and lightguides, two in the front and two in the back of the telescope, define the spatial acceptance for triggers. The crossed scintillators on either side of the beam telescope define a rectangular acceptance window of $20 \mathrm{~mm} \times 10 \mathrm{~mm}$ matching the MIMOSA 26 sensor area. The TLU is based on a commercial Spartan 3 board and features a coincidence unit with discriminator boards accepting up to four PMT input signals. Additionally, it is equipped with several custommade add-on PCBs allowing for an easy integration of user DAQ systems. Providing a programmable logic, the TLU takes a trigger decision based on its four input channels. As interface to the beam telescope DAQ and other user DAQs, the TLU provides RJ45 connectors with four LVDS pairs carrying the trigger clock, busy, reset, and trigger signals. The trigger clock and the busy line are inputs to the TLU, whereas the reset and the trigger line are signals produced by the TLU. The trigger signal is distributed to all DAQ systems on the trigger line. A busy signal is accepted by the TLU from each integrated DAQ individually vetoing subsequent triggers as long as the busy signal is high. More details are available in references [13, 14]. In addition, a LEMO interface is available providing trigger and reset outputs and inputs for the busy signal.

Three different handshake modes handling the trigger/busy signals are available, one of them being the no-handshake mode, in which the TLU issues a fixed-length pulse on the trigger line with the busy line being disregarded. In simple handshake mode, the assertion of a trigger is replied by the integrated DAQ systems by asserting a signal on the busy line. The TLU then de-asserts the trigger and waits for the busy line going low. The trigger data handshake mode uses the same scheme as in the simple handshake mode, but additionally 
the current trigger data are transferred on the trigger line: after the trigger has been deasserted, the trigger number is clocked out.

The zero-suppressed hit data generated by the MIMOSA 26 sensors are transmitted over a ribbon cable to the auxiliary boards, which establish the connection to the data concentrator board collecting the data from all six sensors and the trigger/busy lines from the TLU. The signals from the concentrator board are acquired by a COTS ${ }^{2}$ DAQ system built around the National Instrument (NI) PXIe crate architecture. A custom-made firmware running on a Virtex 5 FPGA embedded in a FlexRIO board (PXIe 7962R) acquires and deserialises the 12 serial links - two per sensor - at $80 \mathrm{Mb} / \mathrm{s}$, detects triggers on the trigger line and reads the trigger data, which are the bottom 15 bits of the trigger counter. The resulting data stream of $960 \mathrm{Mb} / \mathrm{s}$ is read by the CPU (PXIe 8130) from the PXIe bus via a DMA channel and processed on-line by software. Data packets on which the TLU has triggered are selected, demultiplexed and actual frames built. The data are then available to the EUDAQ framework (cf. "The EUDAQ data acquisition framework" section), and an event is written to disk in normal handshake mode, only if a trigger has been raised for a certain telescope readout frame. This co-development, i.e. sharing processing tasks between firmware and software, allows for high flexibility for system upgrades. The presented DAQ architecture is able to read six MIMOSA 26 sensors without any dead-time at up to 8680 frames/s. More details are available in references $[15,16]$.

\section{The EUDAQ data acquisition framework}

The modular cross-platform data acquisition framework EUDAQ [17] has been designed and developed to serve as flexible and simple-to-use data taking software for the EUDETtype beam telescopes, allowing for easy integration of other devices. It consists of completely independent modules communicating via TCP/IP enabling a distributed data acquisition with modules running on separate machines. Currently, EUDAQ is designed for synchronous DAQ systems requiring one event per trigger per attached subdetector system before building the global event. Thus, the trigger rate is always limited by the slowest device.

The central interaction point for users with the framework is the Run Control and its graphical user interface (GUI). All other modules connect to the Run Control at startup and receive additional information from there during operation such as the commands for starting and stopping a DAQ run. The GUI provides all controls necessary to the user on shift. Another important user interface is the Log Collector, gathering logging information from all modules and displaying them in one unified logging window. Log files are stored along with the recorded data files for later reference.

The actual detector data are delivered to the framework by so-called Producers. Producers are the links between the EUDAQ framework and the subdetector systems such as the beam telescope, the TLU, or user DAQ systems. They interface with the EUDAQ library and provide a set of commands to be called by the Run Control. This simple interface scheme eases the integration of user DAQ systems into the software framework. The data read out from the detectors by the individual Producers are sent to the so-called Data Collector. This Data Collector is responsible for the event building, i.e. the correlation of events from all subdetector systems to single global events comprising all data belonging to one trigger. Basic sanity checks such as testing the consistency of event numbers reported by the individual subdetectors are executed. 
To ensure data quality during data acquisition, the Online Monitor tool is available. It connects to the Data Collector requesting a fixed fraction of the recorded events (e.g. one out of a hundred) to fully decode all subdetector data and build basic plots such as hit maps or correlation plots. Thereby, data quality monitoring verifying that the different devices are synchronized in time and all within the geometrical trigger acceptance, is possible during data taking.

The data decoding is performed using Data Converter plug-ins for every detector type attached to the Run Control. The plug-in to be called for a specific subevent is deduced from the event type transmitted by the Producer and written to the data stream by the Data Collector. Each Data Converter plug-in can implement several data format end points allowing, amongst others, for the conversion to the internal EUDAQ format for the Online Monitor, to simple ROOT trees, or to LCIO which is used by the offline reconstruction software described in the following section.

Configuration of the data acquisition framework is performed via global configuration files. The information for every individual module is parsed and distributed by the Run Control. The configuration file is a plain text file divided into sections for the individual modules. Each section contains a list of possibly many (parameter,value)-pairs, which are defined and used by the modules. The full content of the configuration file including commented lines is stored in the so-called Begin-Of-Run Event (BORE) of every run and is thus available later for offline analysis and reference. This greatly simplifies book keeping of detector parameters during test beam shifts since all settings are stored automatically.

\section{Offline analysis and reconstruction using EUTelescope}

For offline analysis and reconstruction of telescope test beam data the EUTelescope software package $[18,19]$ is available and features a close integration of the EUDAQ software framework described in "The EUDAQ data acquisition framework" section. EUTelescope is based on the ILCSoft framework [20] which provides the basic building blocks for offline analysis such as a generic data model (Linear Collider I/O, LCIO), a geometry description language (GEAR) and the central event processor (Marlin) [21].

Marlin allows for a modular composition of analysis chains for various applications. Every task is implemented as an independent processor which is called by Marlin for every event. Each processor exposes a set of parameters to the user which can be configured and loaded at runtime via so-called steering files in XML format. This way the Marlin/Processor architecture gives maximum flexibility to the user.

EUTelescope provides several processors for Marlin, implementing algorithms necessary for a full track reconstruction and data analysis of test beam experiments. Figure 3 shows the analysis strategy of the framework starting from the recorded detector response to the final reconstructed particle tracks. At low-energy beam lines such as the DESY-II test beam facility, multiple scattering is an important contribution to the overall track resolution uncertainty, especially in measurements with non-negligible DUT material budget, cf. the "GBL track fits, multiple scattering and residuals" subsection. Therefore, EUTelescope provides processors implementing advanced algorithms for tracking based on the concepts of a Deterministic Annealing Filter (DAF) [22] or General Broken Lines (GBL) [23, 24], which account for scattering in all material present in the beam. For high-energy beam lines a simple straight line fit provides sufficient precision and a maximum of computational performance by employing a $\chi^{2}$-minimisation 


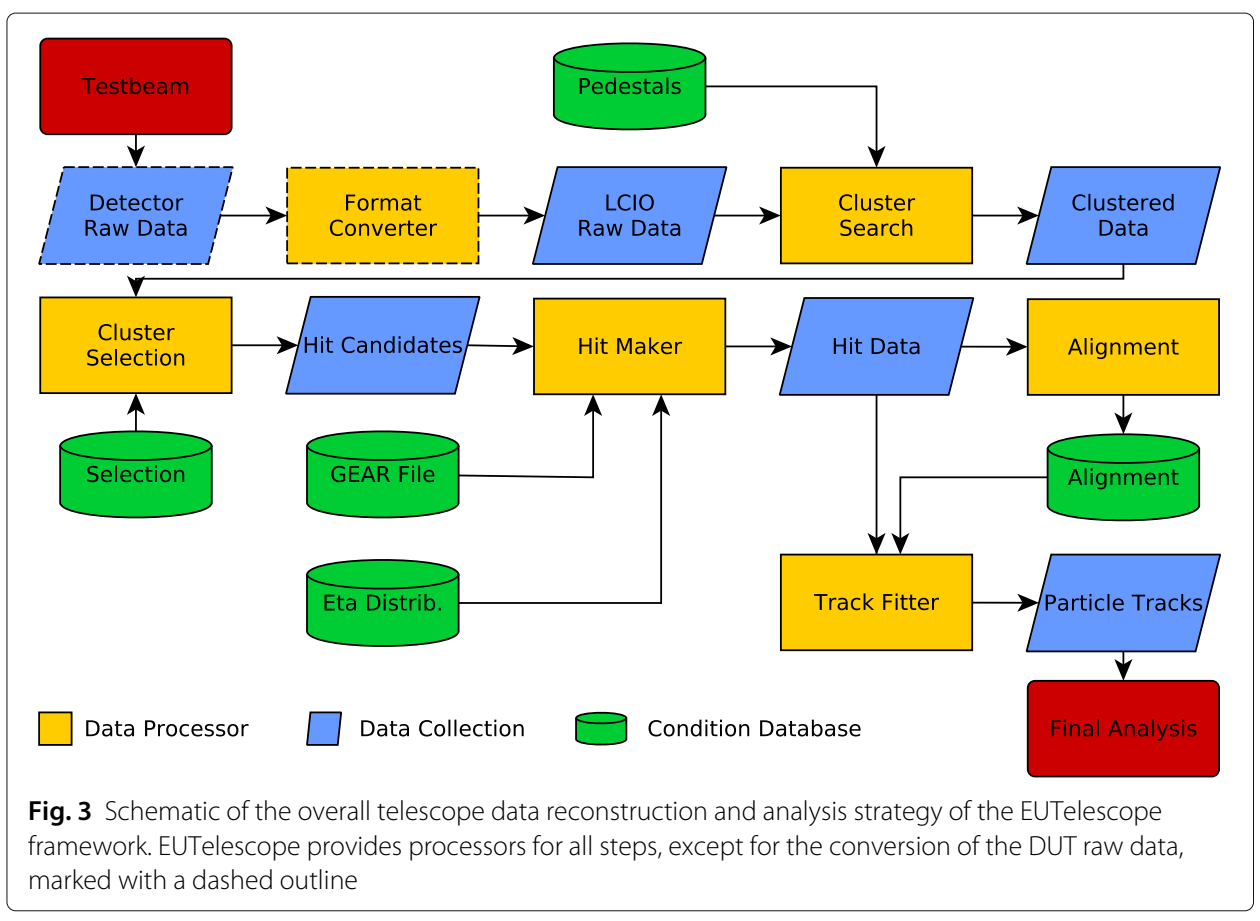

method $[25,26]$ to calculate the track parameters. In addition, precise offline detector alignment can be performed by minimising track residuals using the EUTelescope alignment processor which utilises the Millepede-II algorithm [27].

EUTelescope comes with its own job submission framework Jobsub that allows for submission of analysis jobs on local machines or on larger batch computing clusters such as the $\mathrm{NAF}^{3}$ or LXPLUS ${ }^{4}$ for bulk reconstruction. Using its flexible configuration file concept and the global run database storing user defined variables, Jobsub eases the implementation of per-run variables for reconstruction such as beam energy or detector alignment.

\section{Track resolution studies}

The data presented were taken in 2015 using DATURA, an EUDET-type beam telescope, which was operated with EUDAQ, cf. the "Components of the EUDET-type beam telescopes" and "The EUDAQ data acquisition framework" sections. Results shown in this section have been obtained using the EUTelescope software described in the "Offline analysis and reconstruction using EUTelescope" section. The set-up does not include any additional DUT, only six MIMOSA 26 telescope planes. Track fits are performed using the GBL formalism and track residuals, i.e. the distance between the track fit and the measured hit, are calculated for each telescope plane. The intrinsic resolution of the sensor planes is derived from data and used as input for track resolution predictions.

\section{Data analysis flow and pattern recognition}

After conversion from raw MIMOSA 26 data to the LCIO data format, a hot pixel search is performed marking pixels with firing frequencies above a certain threshold. Clusters are formed from fired, adjacent pixels and translated from two-dimensional entities on the individual telescope planes into hits in a global three-dimensional reference frame. 
Clusters containing at least one hot pixel are removed from the analysis. Triplets are built from hits in the three upstream and three downstream planes separately: First, doublets are defined by straight lines from all hits in plane 0 to all hits in plane 2. A valid triplet is found, if a matching hit in plane 1 is present within a matching distance of $100 \mu \mathrm{m}$, non-valid triplets are discarded. Triplet isolation is ensured by rejecting all triplets whose extrapolation at a chosen $z$-coordinate approaches other triplet extrapolations closer than $300 \mu \mathrm{m}$, the $z$-coordinate being the centre between plane 2 and plane 3. The procedure is repeated for the downstream planes. Matching triplets from the up- and downstream planes are identified by isolated triplets that intersect within a radius of $100 \mu \mathrm{m}$, again at the centre between plane 2 and plane 3. In turn, GBL tracks are formed by the six hits belonging to the matched triplets. For offline alignment, the GBL tracks are passed to Millepede-II in order to determine shift and rotation alignment constants for every telescope plane. Alignment constants of the sensitive coordinates are determined to submicron precision and are hence much smaller than the intrinsic resolution of the detectors used. After applying the alignment corrections, the above track finding is repeated, and GBL tracks are calculated based on the corrected hits. These tracks can be formed in a biased or in an unbiased way, where biased tracks include the measured hit information of every plane in contrast to unbiased tracks, that omit the hit information of the plane under investigation. Finally, a fiducial cut discards tracks at the border of the sensors and a cut on the $\chi^{2}$-probability for the given number of degrees of freedom rejects track with outliers from the further analysis.

\section{GBL track fits, multiple scattering and residuals}

The figure of merit for a beam telescope is its track resolution ${ }^{5} \sigma_{\mathrm{t}, \mathrm{b}}\left(\sigma_{t u}\right)$. It defines the precision with which a particle trajectory can be determined for a biased (unbiased) track. This quantity is a function of the track path, i.e. it is not constant along the trajectory. It depends on the intrinsic resolution $\sigma_{\text {int }}$ of the sensors, that have been used to measure the hits belonging to the track, the number of measurements and their positions as well as the amount of multiple scattering of the beam particles.

Various methods exist to perform the calculation of the track resolution. In the formalism of GBL, an initial simple trajectory (straight line, helix) is corrected for multiple scattering. A single, global $\chi^{2}$-fit of a track model is performed involving all measured hits including their measurement uncertainties (intrinsic resolution) and scattering angle uncertainties along the trajectory. For tracks not bent by a magnetic field, this results in trajectories formed of straight lines between defined scatterers allowing for kinks only at the scatterers themselves. The track resolution stems from the covariance matrix, which is calculated for each track parameter [23, 24]. The method of GBL makes various assumptions and simplifications:

- Scatterers, the origin of kinks in the trajectory, are thin, i.e. no spatial offsets within a scatterer are taken into account.

- A thick scatterer, like the air between two planes, is modelled by two thin scatterers.

- Corrections, i.e. the offsets between the initial trajectory and the fitted trajectory, are small and the kink angles are to good approximation linear functions of the these corrections, cf. Eq. (3) in reference [23] and Eq. (4) in [27]. 
- All random variables follow normal distributions.

- Bremsstrahlung effects are not taken into account in the track model.

Multiple Coulomb scattering causes angular deflection of charged particles traversing any medium. The angular scattering distribution is centred around zero and the standard deviation depends on the particle energy, particle type and the radiation length of the matter traversed [28]. An approximation for high energy protons yields a closed form for the variance of the angular distribution. In the transverse plane, the variance for a single scatterer $\varepsilon$ reads [29]

$$
\Theta_{0}^{2}=\left(\frac{13.6 \mathrm{MeV}}{\beta c p} \cdot z\right)^{2} \cdot \varepsilon \cdot(1+0.038 \cdot \ln (\varepsilon))^{2},
$$

with the velocity $\beta c$, the momentum $p$ and the charge number $z$ of the traversing particle. An accuracy of $11 \%$ for all atomic numbers $Z$ is reported for the standard deviation of the angular distribution. For a composition of scatterers $\varepsilon_{i}$, cf. the "Sensors and mechanics" subsection, the variance including all scatterers (i.e. after the last scatterer) involves the sum over the material budgets and hence $\varepsilon$ in Eq. (1) is to be replaced by $\varepsilon=\sum \varepsilon_{i}$. For track fitting (with a Kalman filter or GBL) the variance from all scatterers along the track has to be distributed over the individual scatterers. Using the material fraction as relative weight results in a formula similar to Eq. (1) with the total material budget used in the logarithmic correction

$$
\Theta_{0, i}^{2} \equiv \frac{\varepsilon_{i}}{\varepsilon} \cdot \Theta_{0}^{2}=\left(\frac{13.6 \mathrm{MeV}}{\beta c p} \cdot z\right)^{2} \cdot \varepsilon_{i} \cdot(1+0.038 \cdot \ln (\varepsilon))^{2} .
$$

The amount of multiple scattering is calculated per sensor considering the measured sensor thickness and a $25 \mu \mathrm{m}$ thick Kapton foil on either side of each sensor. The contribution of air between adjacent planes is accounted for as additional scatterers. Equation (2) demonstrates the advantage of using thin sensors, since the standard deviation of the angular distribution increases with the material budget. This is especially important at low-energy beams, such as the DESY-II test beam, as the standard deviation increases towards lower beam energies.

The biased (unbiased) residual defined as a quantity per track is the distance between the measured hit and the biased (unbiased) track fit. The standard deviation of the residual distribution is hereafter denoted as the residual width. With a known or estimated biased track resolution, i.e. all $\sigma_{\text {int }}\left(z_{i}\right)$ are known or estimates exist, the biased residual width for a set of tracks can be expressed for all positions $z=z_{i}$ as

$$
r_{\mathrm{b}}^{2}(z)=\sigma_{\text {int }}^{2}(z)-\sigma_{\mathrm{t}, \mathrm{b}}^{2}(z) .
$$

The biased residual width at a considered plane $i$ decreases with increasing plane distance $\mathrm{d} z$ and towards lower beam energies. These effects stem from the term $\sigma_{\mathrm{t}, \mathrm{b}}$ : qualitatively, the biased track resolution increases, i.e. worsens, with larger lever arms $\mathrm{d} z$, as an uncertainty in the deflection angle is boosted with larger distances. It converges towards the intrinsic resolution in the limit of large $\Theta_{0}$. Hence, the difference between $\sigma_{\text {int }}$ and $\sigma_{\mathrm{t}, \mathrm{b}}$ decreases. This is also true when comparing residuals from the inner planes to those from the outer ones: The worsening track resolution towards the outer planes decreases the difference with respect to $\sigma_{\text {int }}$ and hence the residual width. 
If the hit measurement of a telescope plane at position $z_{i}$ is not included in the fit, the unbiased residual width at these positions reads [6]

$$
r_{\mathrm{u}}^{2}(z)=\sigma_{\text {int }}^{2}(z)+\sigma_{\mathrm{t}, \mathrm{u}}^{2}(z) .
$$

Contrary to a biased residual, the unbiased residual at a considered plane $i$ increases with increasing plane distance $\mathrm{d} z$ and towards lower beam energies. The unbiased track resolution also increases with larger lever arms $\mathrm{d} z$, but is not limited by the intrinsic resolution. Hence, the sum of $\sigma_{\mathrm{int}}$ and $\sigma_{\mathrm{t}, \mathrm{u}}$ increases. Both the biased and the unbiased residual distributions are a function of the track resolution and therefore feature the same dependencies.

The biased pull of a track is defined as the ratio of the biased residual over the predicted biased residual width

$$
\text { pull }_{\mathrm{b}} \equiv p_{\mathrm{b}}=\frac{r_{\mathrm{b}}}{\sqrt{\sigma_{\text {int }}^{2}-\sigma_{\mathrm{t}, \mathrm{b}}^{2}}} .
$$

Assuming normally distributed residuals, the biased pull distributions are normally distributed and centred at zero with a standard deviation of one, $N(0,1)$, if the material and the scattering therein is known and correctly described. A standard deviation differ-

ing from one originates from an under- or overestimated residual prediction $\sqrt{\sigma_{\text {int }}^{2}-\sigma_{t, b}^{2}}$, which in turn stems from an inaccurate estimate of either the intrinsic resolution or the track resolution. The latter is a consequence of inaccurate inputs for the standard deviation $\Theta_{0}$ of the assumed angular distribution or again for the intrinsic resolution.

\section{Methodology and systematics}

As the intrinsic resolution is not known a priori, an initial estimate, $\hat{\sigma}_{\text {int }}$, is used as an input to the GBL track fitting. The intrinsic resolution $\sigma_{\text {int }}$ is assumed to be constant over the active area of the sensor and is averaged over all cluster sizes. The standard deviation of the pull distributions is iteratively used to update the estimate $\hat{\sigma}_{\text {int }}$. The same estimate is used for all six planes. The calculation is repeated for five beam energies between $6 \mathrm{GeV}$ and $2 \mathrm{GeV}$, ten different sensor thresholds and two different geometries: the narrow geometry with $\mathrm{d} z=\mathrm{d} z_{\text {DUT }}=20 \mathrm{~mm}$ and the wide geometry with $\mathrm{d} z=\mathrm{d} z_{\text {DUT }}=150 \mathrm{~mm}$.

This analysis compares results between biased and unbiased track fits. In principal, both choices lead to compatible results if the material in the beam and the deflection due to multiple scattering therein is correctly described. Systematic uncertainties affect the track resolution, the predicted residual width, and finally the derived intrinsic resolution. The systematic uncertainties of the intrinsic resolution are calculated using the same iterative method used for the central value and are estimated based on uncertain beam energy, uncertain standard deviation of the angular distribution and variations in the fit range.

The beam energy is varied by $5 \%$. The original systematic uncertainty of the standard deviation $\Theta_{0}$ of $11 \%$ is experimentally constrained to $3 \%$, as is shown in the "Performance measurements with DATURA" subsection. In order to estimate the effect of the chosen fit range on the derived intrinsic resolution, the fit range is varied up and down by one standard deviation around a centre value of 2 standard deviations. All systematic uncertainty contributions for the two set-ups are listed in Table 1 . The impact of systematic uncertainties on the result is similar between the biased and the unbiased methodology. In addition to the above mentioned contributions per plane, there is a 
Table 1 Systematic uncertainties of the intrinsic resolution listed for biased and unbiased track fits and two geometries at $6 \mathrm{GeV}$ and $2 \mathrm{GeV}$

\begin{tabular}{|c|c|c|c|c|c|c|c|}
\hline & & & \multicolumn{5}{|c|}{$\sigma_{\sigma_{\text {int }}}$ in $\%$} \\
\hline & & & \multicolumn{3}{|c|}{ Per plane } & All planes & \multirow[t]{3}{*}{$\sqrt{\sum\left(x_{i}\right)^{2}}$} \\
\hline & & & $E$ & $\Theta_{0}$ & fit range & $r m s\left(p_{\mathrm{b}}\right)$ & \\
\hline & & & $\pm 5 \%$ & $\pm 3 \%$ & $\pm 1 \mathrm{std}$ & & \\
\hline \multirow[t]{4}{*}{$6 \mathrm{GeV}$} & \multirow[t]{2}{*}{$20 \mathrm{~mm}$} & biased & \multirow{8}{*}{$\begin{array}{l}-0.34 \\
+0.21 \\
-0.43 \\
+0.71 \\
-3.5 \\
+2.9 \\
-4.8 \\
+5.4 \\
-1.56 \\
+1.13 \\
-1.67 \\
+1.21 \\
-10.5 \\
+15.7 \\
-17.5 \\
+24.9\end{array}$} & \multirow{8}{*}{$\begin{array}{l}+0.08 \\
-0.28 \\
+0.44 \\
-0.25 \\
+1.95 \\
-2.60 \\
+2.97 \\
-4.1 \\
+0.65 \\
-1.22 \\
+0.92 \\
-1.10 \\
+10.2 \\
-6.6 \\
+14.9 \\
-15.2\end{array}$} & \multirow{8}{*}{$\begin{array}{l}+1.76 \\
-1.27 \\
-0.93 \\
-1.00 \\
+6.4 \\
-5.4 \\
-5.3 \\
+3.1 \\
+0.23 \\
+0.33 \\
-2.15 \\
+1.35 \\
+8.0 \\
+0.82 \\
-23.9 \\
+25.1\end{array}$} & 1.57 & 2.6 \\
\hline & & unbiased & & & & 1.23 & 1.8 \\
\hline & $150 \mathrm{~mm}$ & biased & & & & 1.51 & 7.9 \\
\hline & & unbiased & & & & 0.75 & 8.7 \\
\hline \multirow[t]{4}{*}{$2 \mathrm{GeV}$} & $20 \mathrm{~mm}$ & biased & & & & 3.1 & 3.7 \\
\hline & & unbiased & & & & 1.94 & 3.1 \\
\hline & $150 \mathrm{~mm}$ & biased & & & & 0.82 & 20.3 \\
\hline & & unbiased & & & & 1.03 & 38.5 \\
\hline
\end{tabular}

systematic uncertainty of the method itself, as it predicts slightly different intrinsic resolutions for each plane, which changes between geometries, i.e. it is not dominantly caused by an actual difference in intrinsic resolution. The impact of the variance between the width of the pull distributions for different sensors on the intrinsic resolution is therefore included in the table. The most accurate measurement of the intrinsic resolution can be performed at minimal multiple scattering, i.e. at $6 \mathrm{GeV}$ and $\mathrm{d} z=20 \mathrm{~mm}$, featuring a total systematic uncertainty of $2.6 \%$ and $1.8 \%$ for biased and unbiased track fits, respectively. Measurements at $2 \mathrm{GeV}$ and $\mathrm{d} z=150 \mathrm{~mm}$ yield an uncertainty of 20 to $40 \%$.

The assumptions made in the GBL formalism might be less valid for measurements towards lower energies and larger plane spacings, i.e. measurements with a large amount of multiple scattering. Therefore, the predicted track resolution is systematically affected, and hence the pull distributions. Consequently, the estimator $\hat{\sigma}_{\text {int }}$ does not converge towards the true $\sigma_{\text {int }}$. Therefore, a global energy dependent tuning parameter $\kappa$ is introduced, that allows for an adapted amount of scattering used as input to GBL,

$$
\Theta_{\text {corr }}=\kappa(E) \cdot \Theta_{0} .
$$

This parameter is assumed to be constant as a function of the sensor threshold and chosen geometry. Since the intrinsic resolution is a priori independent of the plane spacing $\mathrm{d} z$, a comparison at two precisely measured spacings allows for a calibration of the amount of scattering used as input to GBL at a given beam energy. The value $\kappa$ is determined iteratively under the constraint that the resulting intrinsic resolution for the two geometries agree within $10 \mathrm{~nm}$. The uncertainty of $\kappa$ is defined as the range for which the intrinsic resolution of the wide geometry equals the central value plus/minus one standard deviation of the narrow geometry excluding the uncertainty on $\Theta_{0}$. If $\kappa$ differs significantly from one and/or depends on the beam energy is to be determined with data in the "Performance measurements with DATURA" subsection. Also, a possible difference between the biased and the unbiased fit method is investigated.

\section{Performance measurements with DATURA}

Measurements of particle trajectories were performed to verify the performance of the DATURA telescope at various beam energies, sensor thresholds, and sensor spacings. The residual and pull distributions have been obtained for every sensor plane as 

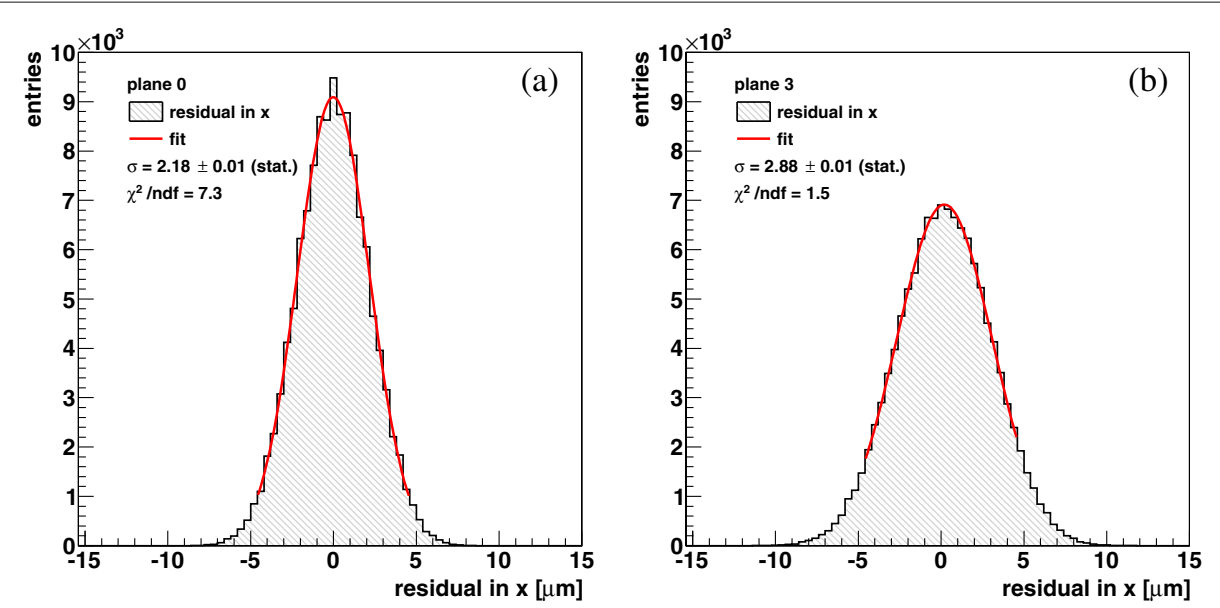

Fig. 4 Biased residual distributions measured with the DATURA telescope at $6 \mathrm{GeV}$ with a plane spacing of $\mathrm{d} z=20 \mathrm{~mm}$. The measured residuals in the $x$-direction for plane $0(\mathbf{a})$ and plane $3(\mathbf{b})$ are shown

described in the "Data analysis flow and pattern recognition", the "GBL track fits, multiple scattering and residuals", and the "Methodology and systematics" subsections for both methodologies, biased and unbiased track fits [30].

Figure 4 shows the biased residual distribution in $x$ for plane 0 and plane 3 for a telescope sensor spacing of $\mathrm{d} z=20 \mathrm{~mm}$, a beam energy of $6 \mathrm{GeV}$ and a sensor threshold setting of $\xi_{\mathrm{n}}=6$. According results for the $y$-direction, further planes, the wide geometry and the unbiased methodology are omitted. The distributions are fitted with a Gaussian, from which the residual width $r_{\mathrm{b}}$ is determined. For plane 3 , the biased residual width in the $x$-direction is

$$
r_{\mathrm{b}}=(2.88 \pm 0.01 \text { (stat.) } \pm 0.08 \text { (sys.) }) \mu \mathrm{m} .
$$

As expected, cf. the "GBL track fits, multiple scattering and residuals" subsection, the biased residual width for the outer plane 0 is smaller than the width obtained from plane 3 . Since the systematic uncertainty dominates the total uncertainty, the statistical contribution is neglected for the further analysis. It should be noted that the residuals feature non-Gaussian tails, as is expected from the underlying physics of the scattering mechanism [29]. The measured residual width used in this work is defined as the standard deviation of a Gaussian fit on the centre $95.5 \%$ of the residual distribution.

The six measured residual widths in the $x$-direction at a beam energy of $6 \mathrm{GeV}$ and a sensor threshold $\xi_{\mathrm{n}}=6$ for both the narrow plane spacing of $\mathrm{d} z=20 \mathrm{~mm}$ and the wide spacing of $\mathrm{d} z=150 \mathrm{~mm}$ are shown in Fig. $5 \mathrm{a}$ and $\mathrm{b}$, respectively. The two distributions show a global maximum in the centre with decreasing residual widths towards both ends. At a considered plane $i$ the residuals are smaller for the wide configuration in comparison to the narrow one, cf. the "GBL track fits, multiple scattering and residuals" subsection.

As described in the "Methodology and systematics" subsection, the tuned amount of multiple scattering used as input to GBL is determined by varying $\kappa$ iteratively until the resulting intrinsic resolution for the two considered geometries agree. This procedure results in an estimate of both $\kappa$ and the true intrinsic resolution. At $6 \mathrm{GeV}$ and $2 \mathrm{GeV}$, a value of $\kappa(6 \mathrm{GeV})=0.76 \pm 0.04$ and $\kappa(2 \mathrm{GeV})=0.75 \pm 0.02$ is found, the latter corresponding to a relative uncertainty of $3 \%$, which is used as the uncertainty on $\Theta_{0}$. The 

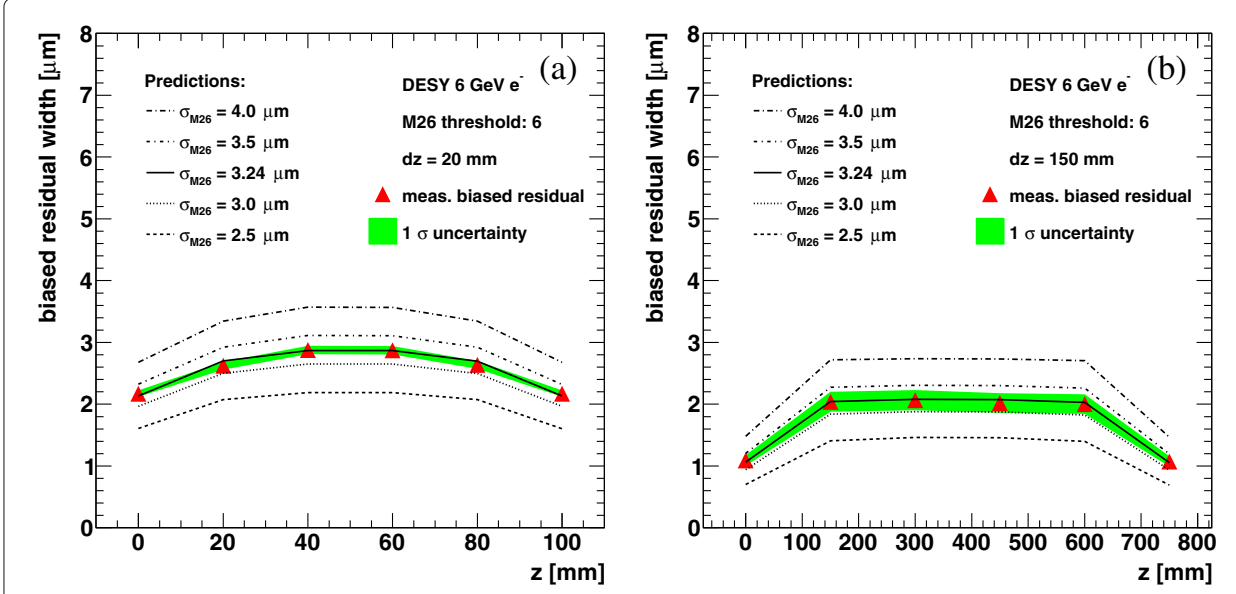

Fig. 5 The measured residual widths of each telescope plane are shown in the $x$-direction for a plane spacing of $\mathrm{d} z=20 \mathrm{~mm}(\mathbf{a})$ and $\mathrm{d} z=150 \mathrm{~mm}(\mathbf{b})$. The black line shows the predicted residual width at $\sigma_{\mathrm{M} 26}=3.24 \mu \mathrm{m}$, the green band the measurement's standard deviation

systematic uncertainty on $\kappa$ includes uncertainties on the beam energy, the fit range and the $\operatorname{rms}\left(p_{\mathrm{b}}\right)$, cf. Table 1 . Additionally, the average intrinsic resolution of the MIMOSA 26 sensors at $6 \mathrm{GeV}$ and threshold $\xi_{\mathrm{n}}=6$ results to

$$
\sigma_{\mathrm{M} 26}=(3.24 \pm 0.09) \mu \mathrm{m}
$$

This average is taken over all twelve measurements of the telescope (two dimensions per plane). The uncertainty is purely of systematic nature and is extracted using Table 1 . The result implies that the average cluster contains more than one pixel and that charge sharing is the underlying cause for an intrinsic resolution better than the binary one. Indeed, the average clustersize at $\xi_{\mathrm{n}}=6$ is about 3.3 pixel. The result is consistent with earlier analyses, which yielded intrinsic resolutions of $\sigma_{\text {int }} \approx 3.4 \mu \mathrm{m}$ [31] and $\sigma_{\text {int }} \approx 3.5 \mu \mathrm{m}$ [11].

The above method is repeated for different beam energies at a fixed threshold $\xi_{\mathrm{n}}=6$. This allows for the determination of $\kappa$ as a function of the beam energy. The correlation is shown in Fig. 6a for both biased and unbiased track fits. For biased track fits, $\kappa$ is found to be constant at a value of $\kappa=0.753 \pm 0.013$ in the energy range covered, which translates to a tuned input of about $-25 \%$ with respect to Eq. (2). This value of $\kappa$ has also been used for the predictions in Fig. $5 \mathrm{a}$ and b. For unbiased track fits, $\kappa$ slightly increases from 0.87 at $2 \mathrm{GeV}$ to 0.94 at $6 \mathrm{GeV}$ with a precision of a single measurement of about $2 \%$. Figure $6 \mathrm{~b}$ shows the measured intrinsic resolution for both methodologies as a function of the beam energy. At each measured energy, they agree within less than one standard deviation over the entire energy range. The averaged absolute values vary slightly with energy, from $3.26 \mu \mathrm{m}$ at $6 \mathrm{GeV}$ to $3.40 \mu \mathrm{m}$ at $2 \mathrm{GeV}$.

Furthermore, the method has been repeated for various sensor thresholds. The threshold applied to each telescope sensor is a critical parameter for the telescope performance. A higher threshold reduces the amount of fired pixels which results in smaller and fewer clusters found on average on a plane and thus limiting the number of reconstructible tracks. This reduces the telescope's triplet efficiency, which is defined as the ratio of 

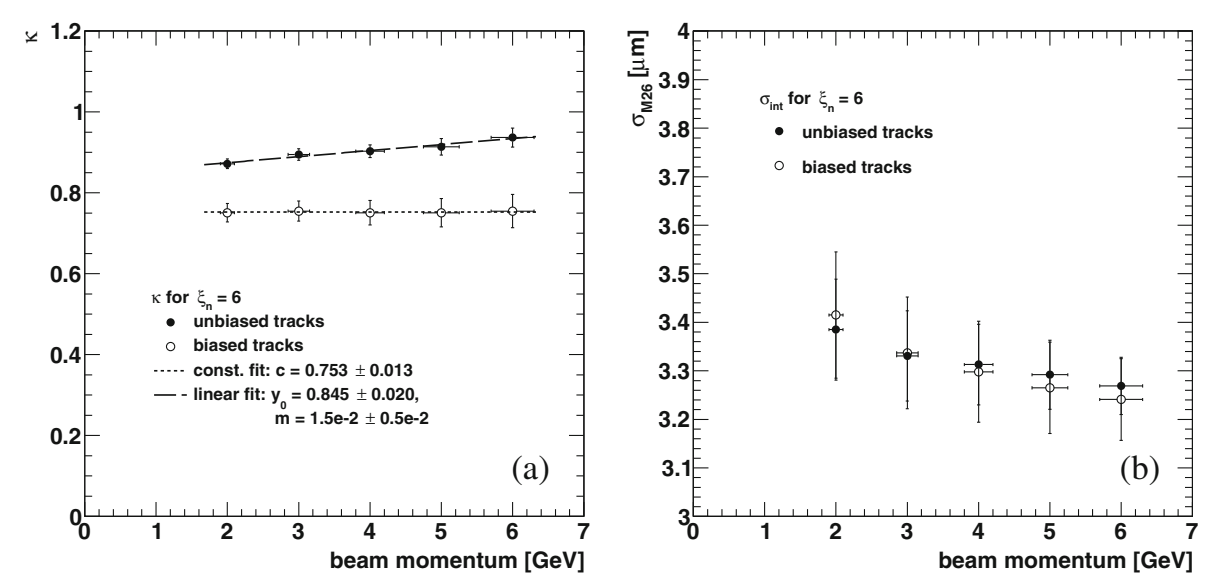

Fig. 6 (a) The global tuning factor $\boldsymbol{\kappa}$ is plotted against beam energy. (b) The derived intrinsic resolution for biased and unbiased track fits is plotted against beam energy

isolated, upstream triplets with a matching hit on plane 3 within an acceptance range $d$ around the triplet extrapolation to the overall number of isolated, upstream triplets

$$
\epsilon_{\text {trip }}=\frac{\text { \#iso., upstream triplets } \cap \text { matching hit on plane } 3}{\text { \#iso., upstream triplets }} \text {. }
$$

A margin $d=50 \mu \mathrm{m}$ is chosen for $6 \mathrm{GeV}$ and $\mathrm{d} z=20 \mathrm{~mm}$, which is scaled with the inverse energy and the plane distance $\mathrm{d} z$ between measurements. In Fig. 7a the telescope triplet efficiency dependence on the sensor threshold is shown for various beam energies and sensor spacings. Statistical uncertainties are negligible. Systematic uncertainties are insignificant due to a precise alignment and the sufficiently large margin $d$. For thresholds $\xi_{\mathrm{n}}=5$ and $\xi_{\mathrm{n}}=6$ the efficiency is measured to $99.6 \%$ and $99.4 \%$, respectively, and the efficiency varies by less than $0.1 \%$ between different geometries and energy settings. With increasing threshold, the efficiency decreases to about $82 \%$ at $\xi_{\mathrm{n}}=12$. Towards lower thresholds, the efficiency also decreases, which seems counter-intuitive, but is related to an increasing number of hot pixels. An observed decrease in efficiency by
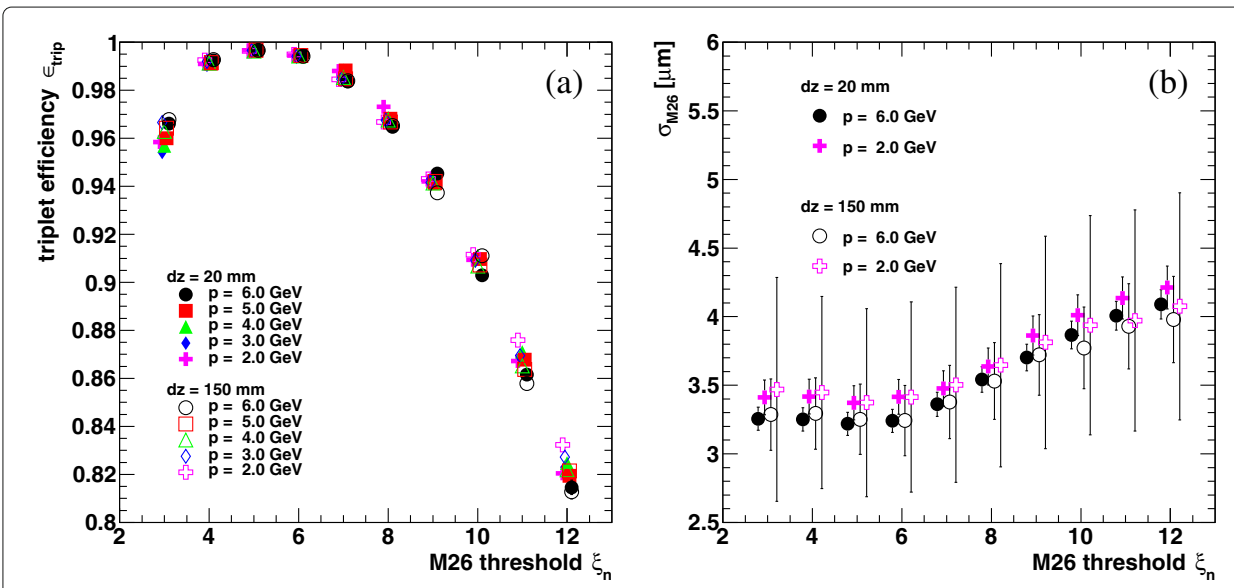

Fig. 7 (a) Triplet efficiency of plane 3 for different beam energies and sensor spacing vs. applied threshold. (b) The measured intrinsic resolution of the $\sigma_{\mathrm{M} 26}$ for different beam energies $p$ and sensor spacing $\mathrm{d} z$ as a function of the applied sensor threshold $\xi_{n}$. In both images values are shifted on the $x$-axis for improved legibility 

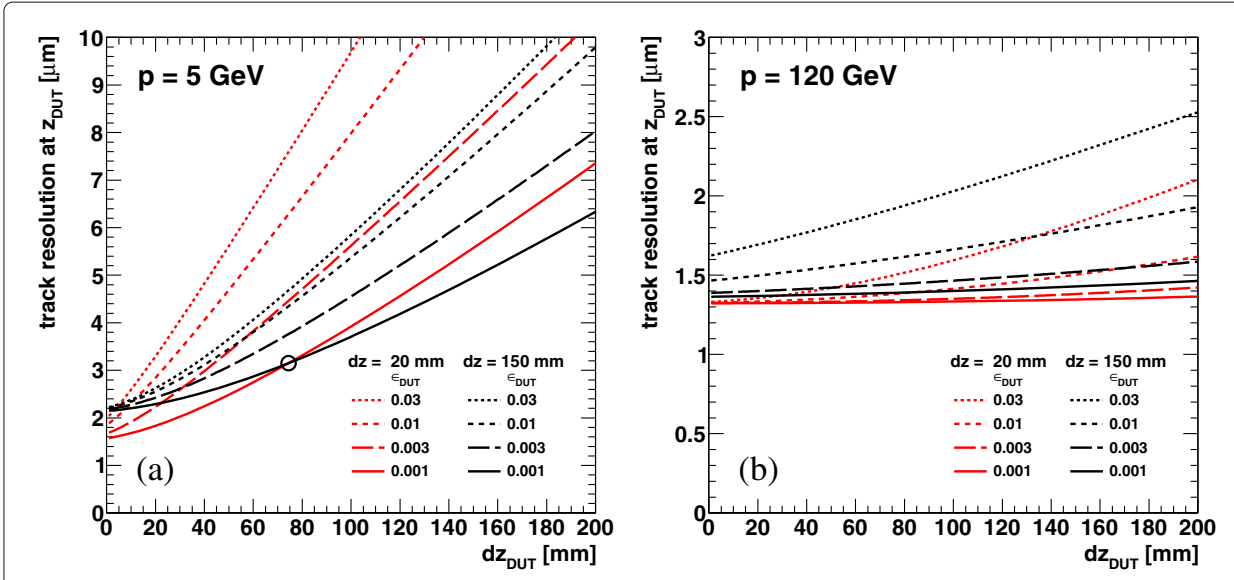

Fig. 8 The track resolution for various material budgets is shown as a function of the equidistant spacing between DUT and neighbouring planes at $5 \mathrm{GeV}(\mathbf{a})$, and $120 \mathrm{GeV}(\mathbf{b})$ at the centre of the beam telescope $\left(z_{\text {DUT }}\right)$

$3.5 \%$ from threshold $\xi_{\mathrm{n}}=5$ to $\xi_{\mathrm{n}}=3$ can be reasonably appointed to the about $1 \%$ hot pixels in a plane at threshold $\xi_{\mathrm{n}}=3$, since all clusters containing at least one hot pixel are removed from the analysis.

The threshold level affects not only the efficiency, but also the intrinsic resolution. With an increased threshold, a hit is formed on average from smaller clusters which deteriorates its position estimate. A deterioration also occurs towards lower thresholds, which allow for an increased number of noise induced signals to pass the zero-suppression on the chip.

Figure $7 \mathrm{~b}$ shows the intrinsic sensor resolutions $\sigma_{\text {int }}$ derived from the biased track fits for different beam energies and plane distances as a function of the applied sensor threshold. An agreement of the various measurements is found within their uncertainties. The minimum of $\sigma_{\text {int }}$ is reached for a sensor threshold setting of $\xi_{\mathrm{n}}=5$. A previous measurement and analysis $[32,33]$ taken at $\xi_{\mathrm{n}}=10$ yielded $\sigma_{\mathrm{M} 26}=(4.35 \pm 0.10) \mu \mathrm{m}$, which seems to slightly overestimate the intrinsic resolution.

\section{Track resolution predictions using General Broken Lines}

Using the measured intrinsic resolution and the material budget in the beam (telescope planes, air, DUT), predictions of the track resolution at the actual DUT position $z_{\mathrm{DUT}}$ (cf. Fig. 2) are rendered possible. Therefore, it is possible to perform an a priori calculation of the optimal telescope geometry for a certain measurement set-up [34]. In this section, $z_{\text {DUT }}$ is assumed to be in the centre between plane 2 and plane 3 .

Using the GBL formalism, the track resolution is analytically calculated at points of interest along the particle trajectory. The track resolution at the DUT for four different DUT material budgets $\varepsilon_{\text {DUT }}$ is depicted as a function of the spacing $\mathrm{d} z_{\text {DUT }}$ in Fig. 8 . Exemplified are two configurations with $\mathrm{d} z=20 \mathrm{~mm}$ and $\mathrm{d} z=150 \mathrm{~mm}$ at (A) DESY-II and (B) SPS beam energies. For $\mathrm{d} z=\mathrm{d} z_{\text {DUT }}=20 \mathrm{~mm}$ at $5 \mathrm{GeV}$ beam energy the track resolution at the DUT is

$$
\sigma_{\mathrm{t}}\left(z_{\mathrm{DUT}}\right)=(1.83 \pm 0.03) \mu \mathrm{m}
$$

for $\varepsilon_{\mathrm{DUT}}=0.001$. For SPS energies, a track resolution of $(1.33 \pm 0.03) \mu \mathrm{m}$ is predicted. The shown resolutions monotonically increase with increasing $\mathrm{d} z$ DUT. In order to 

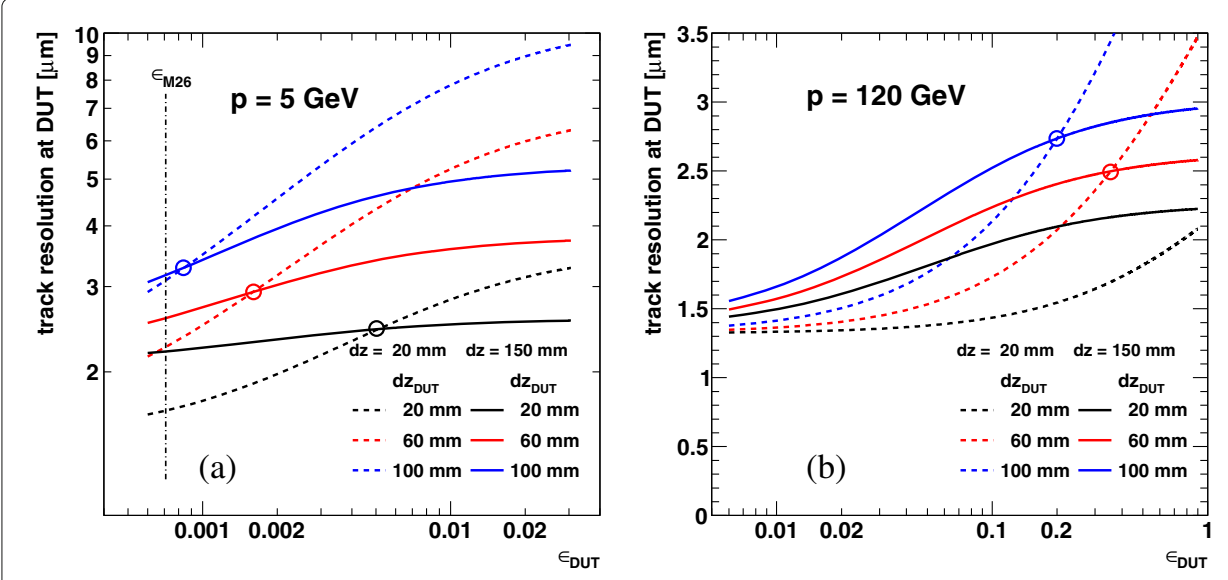

Fig. 9 The calculated track resolutions at the DUT for two geometries are shown as a function of $\varepsilon_{\text {DUT }}$ at $z_{\text {DUT }}$ at a beam energy of $5 \mathrm{GeV}(\mathbf{a})$ and $120 \mathrm{GeV}(\mathbf{b})$. Note the logarithmic scales

achieve the best possible resolution, the inner MIMOSA 26 planes should therefore be positioned as close as possible to the DUT, i.e. $\mathrm{d} z_{\text {DUT }}$ is to be minimised. In addition, Fig. 8a shows, that the optimal plane spacing $\mathrm{d} z$ depends on the actual DUT size along the beam direction and its material budget. For instance for a DUT with a material budget of $\varepsilon_{\text {DUT }}=0.001$ (solid lines) at $5 \mathrm{GeV}$, a narrow configuration shows a better track resolution for $\mathrm{d} z_{\text {DUT }}<74 \mathrm{~mm}$, while a wide configuration is favoured for $\mathrm{d} z_{\text {DUT }}>74 \mathrm{~mm}$. The intersection is marked with a black circle in Fig. 8a. The position of the intersection depends on the material budget of the DUT $\varepsilon_{\text {DUT }}$ and shifts to smaller $\mathrm{d} z_{\text {DUT }}$ with increasing material budget.

At SPS energies, the resolution functions for the wide and the narrow configuration do not intersect, with the narrow configuration showing a slightly better track resolution at the DUT. However, the difference is less than $0.5 \mu \mathrm{m}$ for $z_{\text {DUT }}=100 \mathrm{~mm}$ even at $\varepsilon_{\text {DUT }}=0.03$.

In Fig. 9 the achievable track resolution at $z_{\mathrm{DUT}}$ as a function of the material budget is shown for a beam energy of $5 \mathrm{GeV}$ (A) and $120 \mathrm{GeV}$ (B). Dashed and solid lines represent calculations for $\mathrm{d} z=20 \mathrm{~mm}$ and $\mathrm{d} z=150 \mathrm{~mm}$, respectively. The track resolution deteriorates with increasing $\varepsilon_{\text {DUT }}$. The optimal plane spacing $\mathrm{d} z$ depends on the actual size requirements for the DUT along the beam direction and its material budget. For instance at $5 \mathrm{GeV}$, for a DUT with a material budget of $\varepsilon_{\mathrm{DUT}}=0.002$, a plane spacing of $\mathrm{d} z=20 \mathrm{~mm}$ should be used, if $\mathrm{d} z_{\text {DUT }}$ is as small as $20 \mathrm{~mm}$. However, a plane spacing of $\mathrm{d} z=150 \mathrm{~mm}$ is preferred, if $\mathrm{d} z$ DUT is $60 \mathrm{~mm}$ or larger. Intersections are marked with open circles. At $120 \mathrm{GeV}$, the intersection for $\mathrm{d} z_{\text {DUT }}=100 \mathrm{~mm}$ occurs at a DUT material budget of $\varepsilon_{\mathrm{DUT}}=0.2$, which is considerably larger compared to the case of DESY beam energies. For $\varepsilon_{\text {DUT }}$ below (above) the intersection, a narrow (wide) configuration is preferable. The position of the intersection shifts to smaller material budgets with increasing $\mathrm{d} z_{\text {DUT }}$.

A web tool yielding compatible results in comparison with the GBL calculations is available [35]. The tool calculates track resolutions for a fixed set-up with six planes and one DUT in the centre. A more versatile GBL track resolution calculator allowing for all possible geometries is also available [36]. 


\section{Considerations for DUT integrations}

DUTs can be mechanically integrated into an EUDET-type beam telescope at three positions: upstream or downstream of the telescope, or between the two telescope arms. If placed between the arms, micrometer precision $x y \phi$-stages are available for translation scans and rotation of the DUT [8]. At this position, the maximum width of the DUT set-up is $500 \mathrm{~mm}$. DUTs with larger spatial dimensions are therefore placed at the downstream end of the beam telescope.

User DAQ systems are integrated to the TLU the same way as the beam telescope DAQ itself using either the RJ45 or the LEMO interface, cf. the "Trigger and DAQ system" section. The handshake mode is configurable for each integrated system individually. For the integration of the DUT data stream with EUDAQ, a producer capable of receiving commands by the Run Control and sending data to the EUDAQ Data Collector is necessary as described in "The EUDAQ data acquisition framework" section.

A dedicated alignment run prior to data taking with no DUT in the beam allows for precise alignment of the telescope planes, especially for larger $\varepsilon_{\mathrm{DUT}}$. With a proper alignment at hand, runs including a DUT are to be analysed subsequently in order to align the DUT with respect to the beam telescope. The reconstructed tracks can then be used to characterise the DUT, i.e. to measure its intrinsic resolution or efficiency.

With increasing $\varepsilon_{\mathrm{DUT}}$, and thus multiple scattering within the DUT, the choice of the tracking algorithm needs further consideration. In general, a GBL fit produces tracks with a lower $\chi^{2}$ compared to straight line fits, as kinks at the possibly thick DUT and also at the MIMOSA 26 planes themselves are allowed for. Therefore, using GBL for track fitting is recommended.

For comparison, in a narrow configuration using a $5 \mathrm{GeV}$ electron/positron beam, the track resolution of a EUDET-type beam telescope at a DUT with $\varepsilon_{\mathrm{DUT}}=0.001 \mathrm{using}$ GBL is about $3.7 \mu \mathrm{m}$ making use of all six planes at $\mathrm{d} z=150 \mathrm{~mm}$ and $\mathrm{d} z_{\text {DUT }}=$ $100 \mathrm{~mm}$. The optimal configuration using only the upstream planes is a wide configuration with $\mathrm{d} z=150 \mathrm{~mm}$, and the achievable track resolution is about $8.2 \mu \mathrm{m}$. Hence, in this case, using only the upstream planes deteriorates the resolution by approximately $4.5 \mu \mathrm{m}$.

\section{Conclusion}

In this work the EUDET-type beam telescopes have been described and their performance has been investigated. The highly flexible and versatile EUDET-type beam telescopes come with a Trigger Logic Unit with time stamping capabilities and a clearly defined interface for an integration of user data acquisition systems. It is complemented by a data acquisition system for the telescope sensors, and the powerful software packages EUDAQ and EUTelescope - a modular data acquisition framework and an offline reconstruction software, respectively. The intrinsic resolution of the MIMOSA 26 sensors have been measured to be $(3.24 \pm 0.09) \mu \mathrm{m}$. General Broken Lines calculations predict a track resolution of $(1.83 \pm 0.03) \mu \mathrm{m}$ on the DUT with $20 \mathrm{~mm}$ plane spacing for thin sensors with $\varepsilon_{\text {DUT }}=0.001$ at $5 \mathrm{GeV}$.

Continuous development efforts are ongoing to upgrade and enhance the Trigger Logic Unit, the data acquisition system, and offline reconstruction framework [37]. The maximum achievable rate will be increased by the successor of the TLU. A new plane comprising four MIMOSA 28 planes has been built for the AIDA telescope, covering a 
detection area of $16 \mathrm{~cm}^{2}$, and an appropriate DAQ has been developed to cope with the higher data rates.

Future versions of EUDAQ in combination with a TLU-successor will provide support for parallel data streams and a synchronous mode. The former allows for efficient employment of devices independent of their read-out cycle length and for multiple triggers per read-out cycle. In the synchronous mode, self-triggered devices can be included in the data taking in addition to trigger-based devices. The development of the EUTelescope analysis software is driven by the large and diverse user community. Features currently under development include tracking in magnetic fields and more accurate detector geometry descriptions.

\section{Endnotes}

${ }^{1}$ This is the case for DATURA.

${ }^{2}$ Commercial off-the-shelf

${ }^{3}$ National Analysis Facility. This is a German UNIX cluster.

${ }^{4}$ LXPLUS is a CERN UNIX cluster.

${ }^{5}$ In the literature, this is also referred to as pointing resolution.

\section{Acknowledgements}

We are indebted to Claus Kleinwort for his counsel and numerous discussions. Also, we would like to thank Ulrich Kötz. The test beam support at DESY is highly appreciated. This work is supported by the Commission of the European Communities under the 6th Framework Programme 'Structuring the European Research Area', contract number RII3-026126. Furthermore, strong support from the Helmholtz Association and the BMBF is acknowledged.

\section{Availability of data and materials}

The datasets supporting the conclusions of this article are available from reference [38]. The software used is available from the github repositories: 1) https://github.com/eutelescope/eutelescope, 2) https://github.com/simonspa/ eutelescope/, branch scattering and 3) https://github.com/simonspa/resolution-simulator. For the presented analysis, these specific tags have been used: [30] and [34].

\section{Authors' contributions}

$\mathrm{HJ}, \mathrm{SSP}$ and JDE performed the test beam measurements and hence acquired the data for the presented analysis. HJ elaborated the methodology and systematics and carried out the analysis. DE, TE, JDE and SSP contributed to discussions on methodology and systematics. JB, IR and TE performed preliminary data analyses. SSP and HJ developed the presented simulation tool. HJ, SSP and TE drafted the manuscript. IMG, JB and IR were involved in the commissioning of the first EUDET-type beam telescope. GC proposed the Flex RIO DAQ architecture and coordinated the development of a DAQ demonstrator. MG organised the MIMOSA 26 sensor characterisation activities as well as training sessions on sensor operations. MW coordinated the team of IPHC having designed the MIMOSA 26 sensors and determined its detection performance. CM developed the mechanics and cooling of the EUDET-type beam telescopes. AB and PR developed the core part of the EUTelescope software. JB, IR, HP, TE, SSP and HJ contributed to the EUTelescope software framework. EPC developed the core part of the EUDAQ software framework. DGC, HP, IR, SSP, DH, RP and JDE contributed to the EUDAQ software framework. DGC developed the TLU including its interfaces. DH, RP, HP, IR, SSP, IMG, TE, JDE and HJ contributed to the maintenance and support of the EUDET-type beam telescope. IMG, HP, IR were involved in the overall conceptual design and development of the EUDET-type beam telescope. All authors read and approved the final manuscript.

\section{Competing interests}

The authors declare that they have no competing interests.

\footnotetext{
Author details

${ }^{1}$ Deutsches Elektronen-Synchrotron DESY, Hamburg, Germany. ${ }^{2}$ INFN, Milano, Italy. ${ }^{3}$ IPHC, Strasbourg, France. ${ }^{4}$ DPNC, University of Geneva, Geneva, Switzerland. ${ }^{5}$ University of Bristol, Bristol, UK. ${ }^{6}$ Present Address: Institut für Unfallanalysen, Hamburg, Germany. ${ }^{7}$ EC - Joint Research Centre, Karlsruhe, Germany. ${ }^{8}$ Present Address: SwiftKey (Microsoft), London, UK. ${ }^{9}$ Present Address: SRON, Utrecht, Netherlands. ${ }^{0}$ Present Address: Lund University, Lund, Sweden. ${ }^{11}$ Present Address: CERN, Geneva, Switzerland. ${ }^{12}$ Present Address: CFEL, Hamburg, Germany.
}

Received: 11 May 2016 Accepted: 6 August 2016

Published online: 04 October 2016

References

1. Spannagel S, The CMS collaboration (2014) Test beam campaigns for the CMS Phase I Upgrade pixel readout chip. JINST 9(12):C12001

2. Terzo S, Macchiolo A, Nisius R, Paschen B (2014) Thin n-in-p planar pixel sensors and active edge sensors for the ATLAS upgrade at HL-LHC. JINST 9(12):C12029 
3. Augustin $\mathrm{H}$, et al (2015) The MuPix high voltage monolithic active pixel sensor for the Mu3e experiment. JINST 10(03):C03044

4. Ballabriga R, Campbell M, Heijne E, Llopart X, Tlustos L, Wong W (2011) Medipix3: A 64 k pixel detector readout chip working in single photon counting mode with improved spectrometric performance. Nucl Instrum Methods Phys Rev A 633 Supplement 1(0):S15-S18. 11th International Workshop on Radiation Imaging Detectors (IWORID)

5. Potylitsina-Kube N, de la Taille C, Desch K, Diener R, Gregor IM, Jonsson L, Mnich J, Sefkow F, Timmermans J (2010) EUDET. http://www.eudet.org. Accessed 26 July 2016

6. Bulgheroni A (2009) Results from the EUDET telescope with high resolution planes. Technical Report EUDET-Report-2009-02. https://www.eudet.org/e26/e27/e50990/eudet-report-09-02.pdf. Accessed 21 Apr 2015

7. Hu-Guo C, et al (2010) First reticule size MAPS with digital output and integrated zero suppression for the EUDET-JRA1 beam telescope. Nucl Instrum Methods Phys Rev A 623(1):480-482. 1st International Conference on Technology and Instrumentation in Particle Physics

8. Dreyling-Eschweiler J, Jansen H (2016) Mimosa pixel telescopes. https://telescopes.desy.de/Main_Page. Accessed 17 June 2015

9. Diener R, Meyners N, Potylitsina-Kube N, Stanitzki M (2016) Test Beams at DESY. http://testbeam.desy.de. Accessed 26 July 2016

10. Behnke T, et al (2007) Test Beams at DESY. Technical report. http://www.eudet.org/e26/e28/e182/e283/eudetmemo-2007-11.pdf. Accessed 21 Apr 2015

11. Baudot J, et al (2009) First test results of MIMOSA-26, a fast CMOS sensor with integrated zero suppression and digitized output. In: Nuclear Science Symposium Conference Record 2009. IEEE, Orlando. pp 1169-1173. 10.1109/ NSSMIC.2009.5402399

12. Tsai Y-S (1974) Pair production and bremsstrahlung of charged leptons. Rev Mod Phys 46:815-851

13. Cussans D (2009) Description of the JRA1 Trigger Logic Unit (TLU), v0.2c. Technical report. http://www.eudet.org/ e26/e28/e42441/e57298/EUDET-MEMO-2009-04.pdf. Accessed 21 Apr 2015

14. Cussans D (2007) A trigger/timing logic unit for ILC test-beams. In: Electronics for particle physics. Proceedings, Topical Workshop, TWEPP-07, Prague, Czech Republic, September 3-7, 2007. CERN, Geneva. p 11. http://indico.cern. ch/contributionDisplay. py? contribld $=11 \&$ sessionld $=29 \&$ confld $=11994$

15. Claus G, et al (2010) IPHC \& NI Flex RIO DAQ for EUDET Mimosa 26 Beam Telescope. Technical report. http://www. eudet.org/e26/e28/e86887/e106215/EUDET-Memo-2010-25.pdf. Accessed 21 Apr 2015

16. Claus G, Santos C (2011) Data acquisition system based on NI COTS for pixels sensors characterization in beam test. In: National Instrument Big Physics Symposium. National Instruments, Austin, Texas

17. EUDAQ Software Developers (2016) EUDAQ Website. http://eudaq.github.io. Accessed 21 Apr 2015

18. Rubinskiy I (2010) EUTelescope. Offline track reconstruction and DUT analysis software. Technical Report EUDET-Memo-2010-12. https://www.eudet.org/e26/e28/e86887/e107460/EUDET-Memo-2010-12.pdf. Accessed 21 Apr 2015

19. EUTelescope Software Developers (2016) EUTelescope Website. http://eutelescope.desy.de. Accessed 21 Apr 2015

20. Aplin SJ, Engels J, Gaede F (2009) A production system for massive data processing in ILCSoft. Technical Report EUDET-Memo-2009-12. https://www.eudet.org/e26/e28/e42441/e70141/EUDET-MEMO-2009-12.pdf. Accessed 21 Apr 2015

21. Gaede F, Engels J (2007) Marlin et al - A Software Framework for ILC detector R\&D. Technical Report EUDET-Report-2007-11. https://www.eudet.org/e26/e27/e584/eudet-report-2007-11.pdf. Accessed 21 Apr 2015

22. Gjersdal H, Strandlie A, Røhne O (2014) Straight line track reconstruction for the ATLAS IBL testbeam with the EUDET telescope. Technical Report ATL-INDET-PUB-2014-003. CERN, Geneva

23. Blobel V, Kleinwort C, Meier $F$ (2011) Fast alignment of a complex tracking detector using advanced track models. Comput Phys Commun 182(9):1760-1763. Computer Physics Communications Special Edition for Conference on Computational Physics Trondheim, Norway, June 23-26, 2010

24. Kleinwort C (2012) General broken lines as advanced track fitting method. Nucl Instr Meth Phys Res A 673:107-110

25. Żarnecki A, Nieżurawski P (2007) EUDET Telescope Geometry and Resolution Studies. Technical Report EUDET-Report-2007-01. https://www.eudet.org/e26/e27/e295/eudet-report-2007-01.pdf. Accessed 21 Apr 2015

26. Lutz G (1988) Optimum track fitting in the presence of multiple scattering. Nucl Instr Meth Phys Res A 273(1):349-361

27. Blobel V (2006) Software alignment for tracking detectors 566(1):5-13. Proceedings of the 1st Workshop on Tracking in High Multiplicity Environments

28. Highland V (1975) Some practical remarks on multiple scattering. Nucl Instrum Methods Phys Rev A 129(2):497-499

29. Olive K, et al (2014) Review of Particle Physics. Chin Phys C 38:090001

30. Jansen H, Spannagel S (2016) Analysis Processors for Data taken with EUDET Telescopes. http://dx.doi.org/10.5281/ zenodo. 49065

31. Eichhorn T (2015) Development of, Silicon Detectors for the High Luminosity LHC. PhD thesis. Universität Hamburg, Hamburg. DESY-THESIS-2015-024

32. Behr J (2010) Test Beam Measurements with the EUDET Pixel Telescope. Technical Report EUDET-Report-2010-01. https://www.eudet.org/e26/e27/e107291/eudet-report-2010-01.pdf. Accessed 21 Apr 2015

33. Behr J (2010) Jets at High $Q^{2}$ at HERA and Test Beam Measurements with the EUDET Pixel Telescope. PhD thesis. Universität Hamburg, Hamburg. DESY-THESIS-2010-038

34. Spannagel S, Jansen H (2016) GBL Track Resolution Calculator v2.0. http://dx.doi.org/10.5281/zenodo.48795

35. Kulis S, Dannheim D (2014) Telescope resolution calculator. http://skulis.web.cern.ch/skulis/telescope/. Accessed 21 Apr 2015

36. Spannagel S, Jansen H (2016) GBL Track Resolution Calculator. https://github.com/simonspa/resolution-simulator. Accessed 3 Mar 2016

37. Perrey H (2014) EUDAQ and EUTelescope - software frameworks for testbeam data acquisition and analysis. In: Technology and Instrumentation in Particle Physics. Proceedings of Science, Amsterdam. PoS(TIPP2014)353

38. Jansen H (2016) Dataset for the 'Performance of the EUDET-type beam telescopes' publication (EPJ TI). http://dx.doi. org/10.5281/zenodo.59255 